\title{
A MATHEMATICAL MODEL TO DEMONSTRATE THE SPREAD OF AN EPIDEMIC
}

\author{
Jannatun Nayeem ${ }^{1}$, Kazi Aminur Rahman ${ }^{2}$ and Md. Abu Salek ${ }^{3}$ \\ ${ }^{1}$ Department of Arts and Sciences, Ahsanullah University of Science and Technology, \\ Dhaka, Bangladesh \\ E-mail: salekphd@gmail.com \\ ${ }^{2}$ Department of Mathematics, Dhaka University, Dhaka \\ ${ }^{3}$ Department of Natural Science, United International University, Dhaka-1209, Bangladesh
}

Received 07.04.08 Accepted 30.08.08

\begin{abstract}
Many models for the spread of infectious diseases in populations have been analyzed mathematically and applied to specific diseases. Non-linear dynamical method of projecting the transmission of an epidemic is accurate if the input parameters are reliable. In this paper, a mathematical model is constructed for predicting an epidemic of HIV/AIDS with respect to the presence of infected individuals in the population. For the model, a formula for the basic reproduction number, $R_{0}$ (the expected number of secondary infectious caused by a single new infective introduced into a susceptible population) is determined. The six dimensional model is analyzed qualitatively to determine the stability of equilibria. Analysis of this model includes identifying the threshold $R_{0}$ that determines whether the disease dies out or an epidemic occurs.
\end{abstract}

Key words: Non-linear dynamical method, infectious diseases, epidemic stability, HIV/AIDS.

\section{Introduction}

Models developed for infectious diseases, such as measles or influenza, with short infectious periods have usually contained only one infectious stage $[1,7,8]$. However, these models are generally not suitable for the disease AIDS (Acquired Immunodeficiency Syndrome) caused by HIV (Human Immunodeficiency Virus) because the average infectious period from HIV infection to AIDS is approximately 10 years. For the past several decades mathematical principles have had an important role in disease control strategies [11, 1] and will continue to do so in future. Application of the models becomes easier if there are sufficiently accurate data for the variables used and the concerned parameter values. Mathematical models of transmission dynamics of HIV plays an important role in better understanding of epidemiological patterns and methods for disease control as they provide short and long term prediction of HIV and AIDS incidence and its dependence on various factors. The modeling study is also helpful in determining the demographic and economic impact of the epidemic, which in turn helps us to develop reasonable, scientifically and socially sound intervention plans in order to reduce the spread of the infection. Mathematical modeling studies have shown that the 
AIDS epidemic is very sensitive to the human behaviors that spread HIV, including: the amount of risky behavior, the manner in which that risky behavior is distributed in the population and the social network structures within which people practice those risky behaviors. In fact, these models have shown that if we do not understand all three of these factors, then we can not hope to predict and control the spread of HIV and other sexually transmitted diseases $[9,10]$. Mathematical and statistical models can serve as tools for understanding the epidemiology of HIV and AIDS if they are constructed carefully. Here an attempt is made to model the spread of HIV in a comprehensive manner with limited data. We have proposed a mathematical model for predicting an epidemic of HIV/AIDS into the population. We discuss the model using a simple mathematical approach and calculate the basic reproduction number $\left(R_{0}\right)$ for the model to make a decision for the stability of equilibria. In addition we show that $R_{0}$ is the threshold parameter for the local stability of the disease free equilibria (DFE). Analysis of the centre manifold is done to determine the existence and stability of the endemic equilibria (EE) near the threshold, $R_{0}=1$. Numerical simulations have been carried out to delineate the various analytical results as well.

\section{Model formulation}

In order to derive the model equations, first we divide the population into two classes: Male and Female. Denote the populations of those not infected by HIV as $M_{0}, F_{0}$, those infected as $M_{1}, F_{1}$ and those who subsequently develop AIDS as $M_{2}, F_{2}$ for males and females respectively. Here, it is assumed that HIV-infected individuals will clinically progress to AIDS (the advanced stage of HIV infection) with an average incubation period $1 / \eta_{1}$ (for males), $1 / \eta_{2}$ (for females). Infection is assumed to transmit from male to female and vice versa. No other mode of transmission is considered in the model. Females transmit the virus to their male counterparts with a probability of $\beta_{1}$ (at contact rate $c_{1}$ ), while males transmit to females with a probability of $\beta_{2}$ (at contact rate $c_{2}$ ). Males, females enter the susceptible class (susceptible here means those who can get the infection but are not infected yet) at constant rates $\lambda_{1}$ and $\lambda_{2}$ respectively.

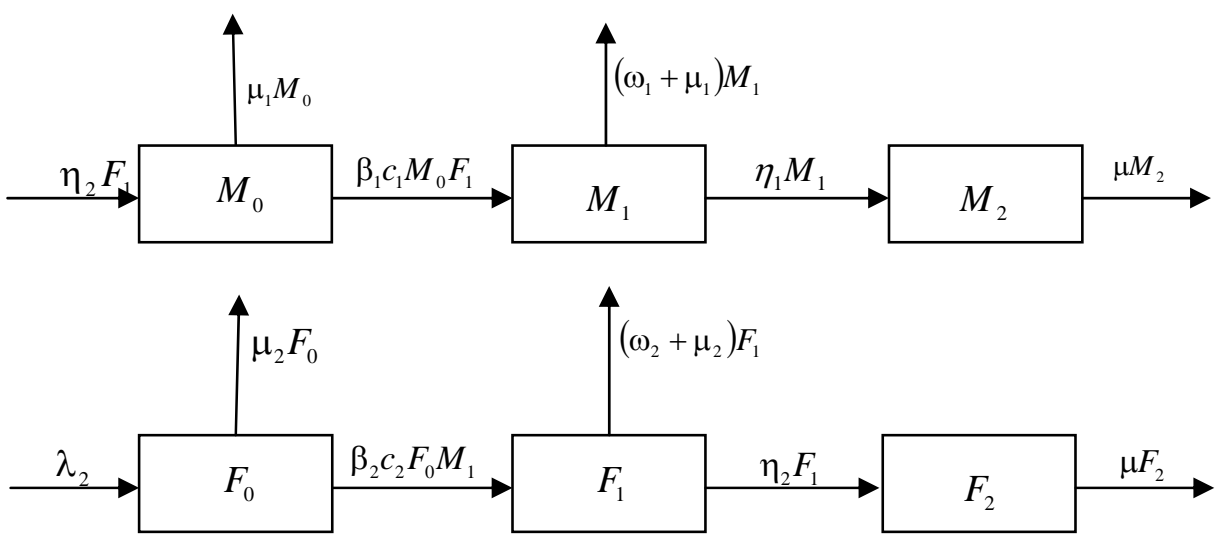

Figure 1: Model structure. The model describes transmission among two classes of people, Male (M) and Female (F). 
Infected individuals are allowed to withdraw from risk behavior (at rates $\omega_{1}$ and $\omega_{2}$, say) and die naturally (at rates $\mu_{1}$ and $\mu_{2}$, say). These death rates are applicable for noninfected individuals. Deaths which are due to AIDS are assumed to be the same (at rate $\mu$, say) for male and female. Here, all the parameters are assumed to be positive. The structure of HIV transmission is summarized in Fig. 1.

The differential equations of the model are given by:

$$
\begin{aligned}
& \frac{d M_{0}}{d t}=\lambda_{1}-\beta_{1} c_{1} M_{0} F_{1}-\mu_{1} M_{0} \\
& \frac{d M_{1}}{d t}=\beta_{1} c_{1} M_{0} F_{1}-\left(\omega_{1}+\eta_{1}+\mu_{1}\right) M_{1} \\
& \frac{d M_{2}}{d t}=\eta_{1} M_{1}-\mu M_{2} \\
& \frac{d F_{0}}{d t}=\lambda_{2}-\beta_{2} c_{2} F_{0} M_{1}-\mu_{2} F_{0} \\
& \frac{d F_{1}}{d t}=\beta_{2} c_{2} F_{0} M_{1}-\left(\omega_{2}+\eta_{2}+\mu_{2}\right) F_{1} \\
& \frac{d F_{2}}{d t}=\eta_{2} F_{1}-\mu F_{2}
\end{aligned}
$$

\section{Stability analysis of disease free equilibria}

\subsection{Disease free equilibrium of the model}

The disease free equilibrium (DFE) for the system (2) is given as

$$
\begin{aligned}
x_{0} & =\left(M_{0}, M_{1}, M_{2}, F_{0}, F_{1}, F_{2}\right)_{D F E} \\
& =\left(\frac{\lambda_{1}}{\mu_{1}}, 0,0, \frac{\lambda_{2}}{\mu_{2}}, 0,0\right)
\end{aligned}
$$

where 0 indicates that there is no infected people (i.e. no disease in the population ).

3.2 Jacobian matrix at DFE and local stability

In order to find the Jacobian at disease free equilibrium we consider the system (2) as

$$
\begin{aligned}
& f_{1}=\beta_{1} c_{1} X_{3} X_{2}-k_{1} X_{1} \\
& f_{2}=\beta_{2} c_{2} X_{4} x_{1}-k_{2} X_{2} \\
& f_{3}=\lambda_{1}-\beta_{1} c_{1} X_{3} X_{2}-\mu_{1} X_{3} \\
& f_{4}=\lambda_{2}-\beta_{2} c_{2} X_{4} X_{1}-\mu_{2} X_{4}
\end{aligned}
$$


where $M_{0}=x_{3}, M_{1}=x_{1}, F_{0}=x_{4}, \quad F_{1}=x_{2}, \quad k_{1}=\left(\omega_{1}+\eta_{1}+\mu_{1}\right)$ and $k_{2}=\left(\omega_{2}+\eta_{2}+\mu_{2}\right)$. Let us consider $f=\left[f_{1}, f_{2}, f_{3}, f_{4}\right]^{T}$ and $x=\left[x_{1}, x_{2}, x_{3}, x_{4}\right]^{T}$ then the Jacobian matrix at DFE is

$$
\begin{aligned}
J_{0} & =D_{x} f\left(x_{0}\right) \\
& =\left(\begin{array}{cccc}
-k_{1} & \frac{\beta_{1} c_{1} \lambda_{1}}{\mu_{1}} & 0 & 0 \\
\frac{\beta_{2} c_{2} \lambda_{2}}{\mu_{2}} & -k_{2} & 0 & 0 \\
0 & -\frac{\beta_{1} c_{1} \lambda_{1}}{\mu_{1}} & -\mu_{1} & 0 \\
-\frac{\beta_{2} c_{2} \lambda_{2}}{\mu_{2}} & 0 & 0 & -\mu_{2}
\end{array}\right)
\end{aligned}
$$

where the notation $D_{x} f\left(x_{0}\right)$ indicates the partial derivative of $f$ with respect to $x$ evaluated at $x=x_{0}$.

Since all the eigenvalues of the matrix $J_{0}$ have negative real parts, the DFE is locally asymptotically stable.

\section{Existence and stability analysis of endemic equilibria}

4.1 Endemic equilibrium of the model

The model has also an endemic equilibrium (EE). By setting the left hand side of system (2) equal to 0 , the EE of the model is found and given as

$$
\begin{aligned}
x^{*}= & \left(M_{0}, M_{1}, M_{2}, F_{0}, F_{1}, F_{2}\right)_{E E} \\
= & \left(\frac{1}{\mu_{1}}\left(\lambda_{1}-\frac{\mu_{1} A}{B F \beta_{2} c_{2}}-\frac{\omega_{1} A}{B F \beta_{2} c_{2}}-\frac{\eta_{1} A}{B F \beta_{2} c_{2}}\right), \frac{A}{B F \beta_{2} c_{2}}, \frac{\eta_{1} A}{B F \mu \beta_{2} c_{2}},\right. \\
& \left.\frac{1}{\mu_{2}}\left(\lambda_{2}-\frac{\mu_{2} A}{D E \beta_{1} c_{1}}-\frac{\omega_{2} A}{D E \beta_{1} c_{2}}-\frac{\eta_{2} A}{D E \beta_{1} c_{1}}\right), \frac{A}{D E \beta_{1} c_{1}}, \frac{\eta_{2} A}{D E \beta_{1} c_{1}}\right)
\end{aligned}
$$

where,

$$
\begin{aligned}
A= & \lambda_{1} \beta_{1} c_{1} \lambda_{2} \beta_{2} c_{2}-\mu_{1}^{2} \mu_{2}^{2}-\mu_{1} \omega_{1} \mu_{2}^{2}-\mu_{1} \eta_{1} \mu_{2}^{2}-\mu_{1}^{2} \mu_{2} \omega_{2} \\
& -\mu_{1} \omega_{1} \mu_{2} \omega_{2}-\mu_{1} \eta_{1} \mu_{2} \omega_{2}-\mu_{1}^{2} \mu_{2} \eta_{2}-\mu_{1} \omega_{1} \mu_{2} \eta_{2}-\mu_{1} \eta_{1} \mu_{2} \eta_{2} \\
B= & \mu_{1}+\omega_{1}+\eta_{1} \\
D= & \lambda_{1} \beta_{2} c_{2}+\mu_{1} \mu_{2}+\omega_{1} \mu_{2}+\eta_{1} \mu_{2} \\
E= & \mu_{2}+\omega_{2}+\eta_{2} \\
F= & \beta_{1} c_{1} \lambda_{2}+\mu_{1} \mu_{2}+\mu_{1} \omega_{2}+\mu_{1} \eta_{2}
\end{aligned}
$$




\subsection{Jacobian matrix at EE and global stability}

Using the system of equations (3.2)the Jacobian matrix at endemic equilibrium states is given by

$$
\begin{aligned}
J_{1} & =D_{x} f\left(x^{*}\right) \\
& =\left(\begin{array}{cccc}
-k_{1} & \beta_{1} c_{1} M_{0}^{*} & \beta_{1} c_{1} F_{1}^{*} & 0 \\
\beta_{2} c_{2} F_{0}^{*} & -k_{2} & 0 & \beta_{2} c_{2} M_{1}^{*} \\
0 & -\beta_{1} c_{1} M_{0}^{*} & -\left(\beta_{1} c_{1} F_{1}^{*}+\mu_{1}\right) & 0 \\
-\beta_{2} c_{2} F_{0}^{*} & 0 & 0 & -\left(\beta_{2} c_{2} M_{1}^{*}+\mu_{2}\right)
\end{array}\right)
\end{aligned}
$$

where,

$$
\begin{aligned}
& M_{0}^{*}=\frac{1}{\mu_{1}}\left(\lambda_{1}-\frac{\mu_{1} A}{B F \beta_{2} c_{2}}-\frac{\omega_{1} A}{B F \beta_{2} c_{2}}-\frac{\eta_{1} A}{B F \beta_{2} c_{2}}\right) \\
& F_{0}^{*}=\frac{1}{\mu_{2}}\left(\lambda_{2}-\frac{\mu_{2} A}{D E \beta_{1} c_{1}}-\frac{\omega_{2} A}{D E \beta_{1} c_{1}}-\frac{\eta_{2} A}{D E \beta_{1} c_{1}}\right) \\
& M_{1}^{*}=\frac{A}{B F \beta_{2} c_{2}} \\
& F_{1}^{*}=\frac{A}{D E \beta_{1} c_{1}}
\end{aligned}
$$

and the values of $A, B, D, E$ and $F$ are given above in equation $(4.1 b-4.1 f)$.

Since it is not easy to find out the eigenvalues of matrix $J_{1}$ at EE analytically, we use the data from Table 1 and after calculating we have seen that all eigenvalues are negative. So, easily we can say that the endemic solution is also stable.

\section{The basic reproduction number}

The basic reproduction number, denoted $R_{0}$ is "the expected number of secondary cases produced, in a completely susceptible population, by a typical infective individual"[4]. If $R_{0}<1$, then on average an infected individual produces less than one new infected individual over the course of its infectious period, and the infection cannot grow. Conversely, if $R_{0}>1$, then each infected individual produces, on average, more than one new infection, and the disease can invade the population (see the survey paper by Hethcote[6]).

Here, we have found the basic reproduction number using the method discussed in [14]. To find $R_{0}$ the following notation is used: 


$$
\left[\begin{array}{l}
\frac{d x_{1}}{d t} \\
\frac{d x_{2}}{d t}
\end{array}\right]=\left[\begin{array}{l}
F_{1} \\
F_{2}
\end{array}\right]-\left[\begin{array}{l}
V_{1} \\
V_{2}
\end{array}\right]
$$

where,

$$
\left[\begin{array}{l}
F_{1} \\
F_{2}
\end{array}\right]=\left[\begin{array}{l}
\beta_{1} c_{1} X_{3} X_{2} \\
\beta_{2} c_{2} X_{4} X_{1}
\end{array}\right]
$$

and

$$
\left[\begin{array}{l}
V_{1} \\
V_{2}
\end{array}\right]=\left[\begin{array}{l}
k_{1} x_{1} \\
k_{2} x_{2}
\end{array}\right]
$$

Then, letting $y=\left[x_{1}, x_{2}\right]^{T}$, we let $F$ and $V$ be the Jacobian. Now we have

$$
\begin{aligned}
F & =\frac{\partial F}{\partial y} \\
& =\left(\begin{array}{ll}
\frac{\partial F_{1}}{\partial x_{1}}\left(x_{0}\right) & \frac{\partial F_{1}}{\partial x_{2}}\left(x_{0}\right) \\
\frac{\partial F_{2}}{\partial x_{1}}\left(x_{0}\right) & \frac{\partial F_{2}}{\partial x_{2}}\left(x_{0}\right)
\end{array}\right) \\
& =\left(\begin{array}{cc}
0 & \beta_{1} c_{1} x_{3} \\
\beta_{2} C_{2} x_{4} & 0
\end{array}\right)
\end{aligned}
$$

and

$$
\begin{aligned}
V & =\frac{\partial V}{\partial y} \\
& =\left(\begin{array}{ll}
\frac{\partial V_{1}}{\partial x_{1}}\left(x_{0}\right) & \frac{\partial V_{1}}{\partial x_{2}}\left(x_{0}\right) \\
\frac{\partial V_{2}}{\partial x_{1}}\left(x_{0}\right) & \frac{\partial V_{2}}{\partial x_{2}}\left(x_{0}\right)
\end{array}\right) \\
& =\left(\begin{array}{cc}
k_{1} & 0 \\
0 & k_{2}
\end{array}\right)
\end{aligned}
$$

Thus, following reference [14], $F V^{-1}$ is the next generation matrix for the model and

$$
R_{0}=\rho\left(F V^{-1}\right)
$$




$$
\begin{aligned}
& =\left(\frac{\beta_{1} c_{1} \beta_{2} c_{2} x_{3} x_{4}}{k_{1} k_{2}}\right)^{\frac{1}{2}} \\
& =\left(\frac{\beta_{1} c_{1} \beta_{2} c_{2} \lambda_{1} \lambda_{2}}{\mu_{1} \mu_{2}\left(\omega_{1}+\eta_{1}+\mu_{1}\right)\left(\omega_{2}+\eta_{2}+\mu_{2}\right)}\right)^{\frac{1}{2}}
\end{aligned}
$$

where $\rho\left(F V^{-1}\right)$ denotes the spectral radius of the matrix $F V^{-1}$.

Suppose $R_{0}>1$.Then

$$
\begin{aligned}
& R_{0}>1 \\
& \Leftrightarrow\left(\beta_{1} c_{1} \beta_{2} c_{2} \lambda_{1} \lambda_{2}\right)^{\frac{1}{2}}>\left(\mu_{1} \mu_{2}\left(\omega_{1}+\eta_{1}+\mu_{1}\right)\left(\omega_{2}+\eta_{2}+\mu_{2}\right)\right)^{\frac{1}{2}} \\
& \Leftrightarrow \beta_{1} c_{1} \beta_{2} c_{2} \lambda_{1} \lambda_{2}>\mu_{1} \mu_{2}\left(\omega_{1}+\eta_{1}+\mu_{1}\right)\left(\omega_{2}+\eta_{2}+\mu_{2}\right) \\
& R_{0}<1 . \text { Then } \\
& R_{0}<1 \\
& \Leftrightarrow\left(\beta_{1} c_{1} \beta_{2} c_{2} \lambda_{1} \lambda_{2}\right)^{\frac{1}{2}}<\left(\mu_{1} \mu_{2}\left(\omega_{1}+\eta_{1}+\mu_{1}\right)\left(\omega_{2}+\eta_{2}+\mu_{2}\right)\right)^{\frac{1}{2}} \\
& \Leftrightarrow \beta_{1} c_{1} \beta_{2} c_{2} \lambda_{1} \lambda_{2}<\mu_{1} \mu_{2}\left(\omega_{1}+\eta_{1}+\mu_{1}\right)\left(\omega_{2}+\eta_{2}+\mu_{2}\right)
\end{aligned}
$$

If $\quad R_{0}<1$. Then

\section{Analysis of the centre manifold near $x=x_{0}, R_{0}=1$}

In this section we consider the nature of the equilibrium solutions of the disease transmission model near the bifurcation point $x=x_{0}, R_{0}=1[14]$. Since $R_{0}$ is often inconvenient to use directly as bifurcation parameter, we introduce a bifurcation parameter $\theta$. Let $\theta$ be a bifurcation parameter such that $R_{0}<1$ for $\theta<0$ and $R_{0}>1$ for $\theta>0$ and such that $x_{0}$ is a DFE for all values of $\theta$. Let us consider the system

$$
\dot{x}=f(x, \theta)
$$

where $f$ is as described in equation (3.2). The choice of $\theta$ is given below, when the quality $b$ is calculated. The position of the DFE depends particularly on the choice of $\theta$ and the local stability changes at the point $\left(x_{0}, 0\right)$. The results of centre manifold theory [15] are used here to show that there are nontrivial (endemic) equilibria near the bifurcation point $\left(x_{0}, 0\right)$.

We use the notation $J_{0}=D_{x} f\left(x_{0}, 0\right)$ for the partial derivative of $f$ with respect to $x$ evaluated at $x=x_{0}$ and $\theta=0$. Let $v$ and $u$ be the corresponding left and right eigenvectors chosen such that $v J_{0}=0$ and $J_{0} u=0$ with $v u=1$ where $v=\left[v_{1}, v_{2}, v_{3}, v_{4}\right]$ and 
$u=\left[u_{1}, u_{2}, u_{3}, u_{4}\right]^{T}$. Following reference [14], we define

$$
\begin{aligned}
& a=\frac{v}{2} D_{x x} f\left(x_{0}, 0\right) u^{2}=\frac{1}{2} \sum_{i, j, k=1}^{n} v_{i} u_{j} u_{k} \frac{\partial^{2} f_{i}}{\partial x_{j} \partial x_{k}}\left(x_{0}, 0\right) \\
& b=v D_{x \theta} f\left(x_{0}, 0\right) u=\sum_{i, j=1}^{n} v_{i} u_{j} \frac{\partial^{2} f_{i}}{\partial x_{j} \partial \theta}\left(x_{0}, 0\right)
\end{aligned}
$$

For our model, we have $n=4$ and now we find the values of $a$ and $b$. The sign of $a$ as well as the value of $b$ will help in determining the nature of the endemic equilibria near the bifurcation point.

For $i=1$

$$
\begin{aligned}
a_{1} & =\frac{1}{2} \sum_{j, k=1}^{4} v_{1} u_{j} u_{k} \frac{\partial^{2} f_{1}}{\partial x_{j} \partial x_{k}}\left(x_{0}, 0\right) \\
& =\frac{1}{2} v_{1} u_{2} u_{3} \beta_{1} c_{1}+\frac{1}{2} v_{1} u_{2} u_{3} \beta_{1} c_{1} \\
& =v_{1} u_{2} u_{3} \beta_{1} c_{1}
\end{aligned}
$$

Similarly, for $i=2,3,4$ we get $a_{2}=v_{2} u_{1} u_{4} \beta_{2} c_{2}, a_{3}=-v_{3} u_{2} u_{3} \beta_{1} c_{1}$ and $a_{4}=-v_{4} u_{1} u_{4} \beta_{2} c_{2}$ respectively. Thus we have

$$
\begin{aligned}
a & =a_{1}+a_{2}+a_{3}+a_{4} \\
& =v_{1} u_{2} u_{3} \beta_{1} c_{1}+v_{2} u_{1} u_{4} \beta_{2} c_{2}-v_{3} u_{2} u_{3} \beta_{1} c_{1}-v_{4} u_{1} u_{4} \beta_{2} c_{2} \\
& =u_{2} u_{3} \beta_{1} c_{1}\left(v_{1}-v_{3}\right)+u_{1} u_{4} \beta_{2} c_{2}\left(v_{2}-v_{4}\right)
\end{aligned}
$$

In order to find the value of $b$, let me consider $R_{0}$ as a function of $c_{1}$ i.e. $R_{0}=R_{0}\left(c_{1}\right)$ and fix all other parameters $c_{2}, \beta_{i}, \mu_{i}, \lambda_{i}, \omega_{i}, \eta_{i}$. Let $c_{1}^{*}$ be the value of $c_{1}$ such that $R_{0}\left(c_{1}^{*}\right)=1$. Let $\theta=c_{1}-c_{1}^{*}$, then $R_{0}=R_{0}(\theta)$ with $R_{0}(0)=1$.

For $i=1$

$$
\begin{aligned}
b_{1} & =\sum_{j=1}^{4} v_{1} u_{j} \frac{\partial^{2} f_{1}}{\partial x_{j} \partial \theta}\left(x_{0}, 0\right) \\
& =v_{1} u_{2} \beta_{1} x_{3} \\
& =\frac{v_{1} u_{2} \beta_{1} \lambda_{1}}{\mu_{1}}
\end{aligned}
$$

Similarly, for $i=2,3,4$ we get $b_{2}=0, b_{3}=-v_{3} u_{2} \beta_{1} x_{3}=-\frac{v_{3} u_{2} \beta_{1} \lambda_{1}}{\mu_{1}}$ and $b_{4}=0$ respectively. Thus we have 


$$
\begin{aligned}
b & =b_{1}+b_{2}+b_{3}+b_{4} \\
& =\frac{v_{1} u_{2} \beta_{1} \lambda_{1}}{\mu_{1}}+0-\frac{v_{3} u_{2} \beta_{1} \lambda_{1}}{\mu_{1}}+0 \\
& =\frac{u_{2} \beta_{1} \lambda_{1}}{\mu_{1}}\left(v_{1}-v_{3}\right)
\end{aligned}
$$

Now using the condition $v J_{0}=0$ and $J_{0} u=0$, we can find $v$ and $u$ as

$$
v=\left(v_{1}^{*}, \frac{\beta_{1} c_{1} \lambda_{1} v_{1}^{*}}{k_{2} \mu_{1}}, 0,0\right)
$$

and $u=\left(u_{1}^{*}, \frac{\beta_{2} c_{2} \lambda_{2} u_{1}^{*}}{\mu_{2} k_{2}},-\frac{\beta_{1} \beta_{2} c_{1} c_{2} \lambda_{1} \lambda_{2} u_{1}^{*}}{\mu_{1}^{2} \mu_{2} k_{2}},-\frac{\beta_{2} c_{2} \lambda_{2} u_{1}^{*}}{\mu_{2}^{2}}\right)$

Since $R_{0}=1$ i.e. $k_{1} k_{2} \mu_{1} \mu_{2}-\beta_{1} \beta_{2} c_{1} c_{2} \lambda_{1} \lambda_{2}=0$ then $v_{1}=v_{1}^{*} \neq 0$ and $u_{1}=u_{1}^{*} \neq 0$.

Let $u_{1}^{*}=1$. Then the condition $u v=1$ gives

$$
v_{1}^{*}=\frac{1}{Q}
$$

where

$$
Q=1+\frac{\beta_{1} \beta_{2} c_{1} C_{2} \lambda_{1} \lambda_{2}}{k_{2}^{2} \mu_{1} \mu_{2}}
$$

Substituting the values of $v$ and $u$ in equations (6.4) and (6.5), we get

$$
\begin{aligned}
a & =v_{1} u_{2} u_{3} \beta_{1} c_{1}+v_{2} u_{1} u_{4} \beta_{2} c_{2} \\
& =-\frac{\beta_{1}^{2} \beta_{2}^{2} c_{1}^{2} c_{2}^{2} \lambda_{1} \lambda_{2}^{2}}{Q k_{2}^{2} \mu_{1}^{2} \mu_{2}^{2}}-\frac{\beta_{1} \beta_{2}^{2} c_{1} c_{2}^{2} \lambda_{1} \lambda_{2}}{Q k_{2} \mu_{1} \mu_{2}^{2}}
\end{aligned}
$$

and

$$
\begin{aligned}
b & =\frac{u_{2} \beta_{1} \lambda_{1}}{\mu_{1}} v_{1} \\
& =\frac{\beta_{1} \beta_{2} c_{2} \lambda_{1} \lambda_{2}}{Q \mu_{1} \mu_{2} k_{2}}
\end{aligned}
$$

Thus, it is obvious that $a<0$ and $b \neq 0$ and hence by theorem 2 [14] there are locally asymptotically stable endemic equilibria near $x_{0}$ for $0<\theta<\delta$ where $\delta>0$. Therefore, the bifurcation as $R_{0}$ passes through 1 is a forward bifurcation.

Hence, the DFE is locally asymptotically stable if $R_{0}$ is less than one (i.e. $\theta<0$ ), if $R_{0}$ is greater than one then the DFE is unstable and there is a locally asymptotically stable EE near the DFE. 


\section{Numerical computation and discussion}

To illustrate the various theoretical results discussed in this paper, the model is simulated using the parameter values/ranges shown in Table 1 . Choice of numerical values for the model's parameter values is based upon published data on the transmission dynamics of HIV in one of the major cities, Delhi of India [2, 3, 5, 12, 13]. Since AIDS is a sexually transmitted disease, we restrict our analysis to the population that is age 15 or older. Here, we have used MATLAB 6.1 package for plotting the solutions of our model. It is easily observed from Fig. 2 that the final total population size is a lot smaller than the initial population size. So, HIV/AIDS has the potential impact to cause a population crush (i.e. an epidemic occurs) if all the parameter values remain constant.

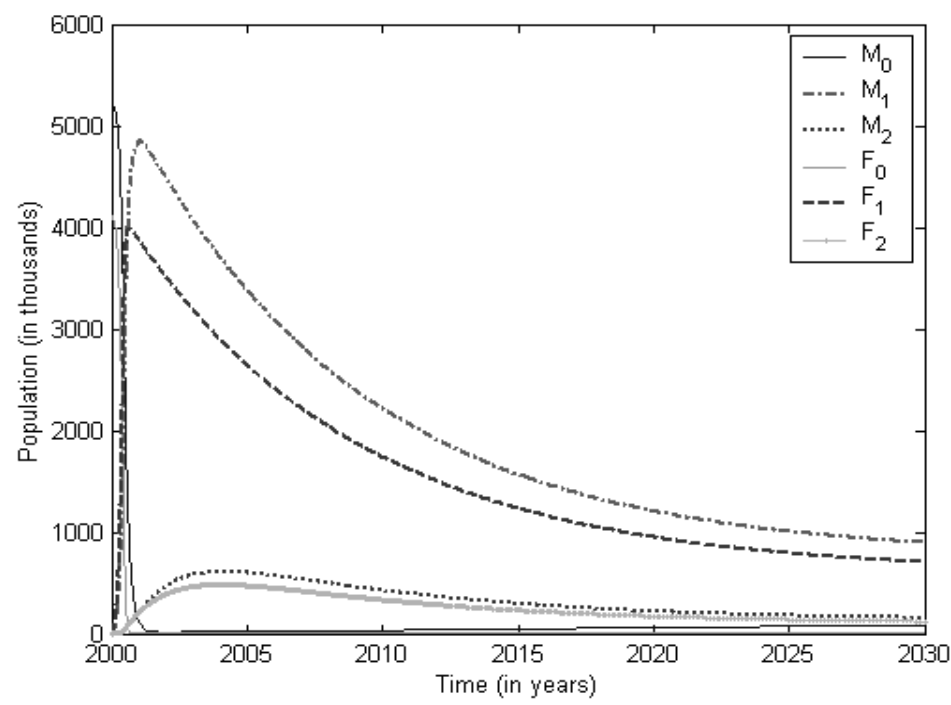

Figure 2: Trends of HIV, AIDS and susceptible population.

The basic reproduction number, $R_{0}$ (given by 5.8) is a threshold parameter for the model. Here, $R_{0}=117.308>1$ and the disease persists limiting to the endemic equilibrium point (EEP). Moreover, the analysis of the centre manifold yields a second parameter, $a$ (given by 6.2) whose sign indicates the existence and stability near $x=x_{0}$ and $R_{0}=1$. Note that $a=-2.08783$ (i.e. negative). Thus for $R_{0}<1$, the DFE is stable where as for $R_{0}>1$, the DFE is unstable and the EEP is stable.

\section{Conclusion}

Application of non-linear differential equations model in predicting the course of epidemics is well established. The model presented here captures the dynamics of virus transmission and full-blown disease in males and females. The model is analyzed to investigate the existence and stability of the associated equilibria. Numerical simulations were carried out using reasonable sets of parameter values to assess the spread and the persistency of HIV/AIDS epidemic in the country. The model shows that there is a possibility of an epidemic if all the parameter values remain constant (i.e. no initiatives 
are taken for changing the behavioural parameters). This model can contribute to the planning of preventive procedures in case of HIV transmission into the population. Behavioural parameters can help public health planning as well.

Table 1: Description and estimation of parameters.

\begin{tabular}{|c|c|c|}
\hline Parameter & Description & Estimation value/range \\
\hline$\lambda_{1}$ & $\begin{array}{l}\text { Recruitment rate of susceptible males into the } \\
\text { community (in thousands) }\end{array}$ & 92.58 (year) \\
\hline$\lambda_{2}$ & $\begin{array}{l}\text { Recruitment rate of susceptible females into the } \\
\text { community (in thousands) }\end{array}$ & 69.95 (year) \\
\hline 1 & Average life expectancy for males & 55.6 years \\
\hline $\begin{array}{c}\mu_{1} \\
1\end{array}$ & & \\
\hline$\frac{1}{\mu_{2}}$ & Average life expectancy for females & 58.8 years \\
\hline$\beta_{1}$ & $\begin{array}{l}\text { Probability of females transmit the virus to their } \\
\text { male counterparts }\end{array}$ & 0.00155 \\
\hline$\beta_{2}$ & $\begin{array}{l}\text { Probability of males transmit the virus to their } \\
\text { female counterparts }\end{array}$ & 0.00105 \\
\hline$c_{1}$ & Contact rate of females & 1.001 \\
\hline$C_{2}$ & Contact rate of males & 5.440 \\
\hline$\omega_{1}$ & Withdrawal rate from risk behavior for males & 0 \\
\hline$\omega_{2}$ & Withdrawal rate from risk behavior for females & 0 \\
\hline$\eta_{1}$ & Incubation period for males & 0.1 year \\
\hline$\eta_{2}$ & Incubation period for females & 0.1 year \\
\hline$\mu$ & Natural death rate & 0.6 \\
\hline
\end{tabular}

\section{REFERENCES}

1. Anderson R M, May R M 1991 Infectious diseases of humans: Dynamics and control (London: Oxford University Press).

2. Census of India 2001, "Report of the technical group on population projections constituted by the national commission on population", (2006).

3. Delhi State AIDS Control Society, Government of Delhi, Population Foundation of India and Population Reference Bureau, "HIV/AIDS in DELHI, Meeting the Challenge”, New Delhi, (2005).

4. Diekmann, O., Heesterbeek, J. A. P. and Metz, J. S. J., "On the definition and the computation of the basic reproduction number ratio $R_{0}$ in models for infectious diseases in heterogeneous populations”, $\mathrm{J}$. Math. Bio. 28, 365-382, (1990).

5. Gisselquist, David and Correa, Mariette, "How much does heterosexual commercial sex contribute to India’s HIV epidemic?” International Journal of STD and AIDS 17: 736-742, 2006.

6. Hethcote, H. W., “The mathematics of infectious disease”, SIAM Rev. 42, 599-653, (2000).

7. H. W. Hethcote, Qualitative analysis of communicable disease models, Math. Biosci. 28: 335-356 (1976).

8. H. W. Hethcote and J. W. Van Ark, Epidemiological models for heterogeneous populations: proportionate mixing, parameter estimation and immunization programs, Math. Biosci. 84: 85-118 (1987). 
9. Hyman, JM, and Stanley, EA. Using Mathematical Models to Understand the AIDS epidemic. Math. Biosci., 90: 415-473, (1988.).

10. Hyman, JM, and Stanley, EA. 1994. A Risk-Based Heterosexual Model for the AIDS Epidemic with Biased Sexual Partner Selection. Modeling the AIDS Epidemic: Planning, Policy and Prediction, Raven Press, Ltd.

11. Murray J D (1989) Mathematical biology (Berlin: Springer-Verlag).

12. Rao, Arni S. R. Srinivasa and Kakehashi, Masayuki, "A combination of differential equations and convolution in understanding the spread of an epidemic”, Sadhana, 29, 305-313, (2004).

13. Rao, Arni S. R. Srinivasa, "Mathematical modeling of AIDS epidemic in India”, Current Science, 84, (2003).

14. Van den Driessche, P. and Watmough, James, "Reproduction numbers and sub-threshold endemic equilibria for compartmental models of disease transmission”, Elsevier (Math. Biosci.), (2002).

15. Wiggins, S., "Introduction to Applied Nonlinear Dynamical Systems and Chaos”, Springer-Verlag, Berlin, (1990). 